\title{
Multi-Objective Optimization of Wire Electro Discharge Machining (WEDM) Process Parameters Using Grey-Fuzzy Approach
}

\author{
Partha Protim Das ${ }^{1 *}$, Sunny Diyaley¹, Shankar Chakraborty², Ranjan Kumar Ghadai \\ 1 Department of Mechanical Engineering, Sikkim Manipal Institute of Technology, Majitar, Sikkim, India \\ 2 Department of Production Engineering, Jadavpur University, Kolkata, India \\ * Corresponding author, e-mail: parthaprotimdas@ymail.com
}

Received: 01 March 2018, Accepted: 21 September 2018, Published online: 16 November 2018

\begin{abstract}
Wire electro discharge machining (WEDM) is a versatile non-traditional machining process that is extensively in use to machine the components having intricate profiles and shapes. In WEDM, it is very important to select the optimal process parameters so as to enhance the machine performance. This paper emphasizes the selection of optimal parametric combination of WEDM process while machining on EN31 steel, using grey-fuzzy logic technique. Process parameters such as servo voltage, wire tension, pulse-on-time and pulse-off-time were considered while taking into account several multi-responses such as material removal rate (MRR) and surface roughness $(S R)$. It was found that pulse-on-time of $115 \mu \mathrm{s}$, pulse-off-time of $35 \mu \mathrm{s}$, servo voltage of $40 \mathrm{~V}$ and wire tension of $5 \mathrm{kgf}$ results in a larger value of grey fuzzy reasoning grade (GFRG) which tends to maximize MRR and improve $S R$. Finally, analysis of variance (ANOVA) is applied to check the influence of each process parameters in the estimation of GFRG.
\end{abstract}

Keywords

WEDM, machining parameters, multi-objective optimization, Grey-fuzzy logic, ANOVA

\section{Introduction}

Non-traditional machining (NTM) processes are being extensively used in the automobile, dies, aerospace and tool making industries which strictly aim for high accuracy and surface finish irrespective of its hardness. WEDM, an electro-thermal metal removal process, is widely being used in automotive, aerospace and nuclear industries, to machine irregular shapes, precise and complex designs in various electrically conductive difficult-to-machine materials. WEDM is a unique class of traditional electrical discharge machining (EDM) process, where the electrode in form of a wire (made of thin brass, copper, or tungsten of diameter $0.05-0.3 \mathrm{~mm}$ ) which moves continuously. The movement of the electrode is controlled numerically to obtain the desired shape, size and accuracy of the workpiece. The wire is kept in tension to avoid inaccurate shapes by means of a mechanical device. Material removal in WEDM process takes place by means of erosion resulting from repetitive, rapid and discrete spark discharges between the wire and the workpiece while being immersed in a dielectric fluid (deionized water/kerosene). The spark produced melts and vaporizes a small portion of workpiece which are then flushed away by dielectric fluid [1]. It has several machining parameters such as ignition pulse current, applied voltage, idle time, pulse duration, servo speed, wire speed, wire tension and injection pressure for dielectric, which adversely affects its performance measures such as $M R R$, cutting speed, and $S R$ etc. [2]. Machining parameters plays an important role in obtaining high precision machining with quality responses. The selection of these machining parameters is vital as improperly selected parameters might result in serious consequences like wire breakage, short-circuiting of wire. Hence, there is a demand for research that could generate a systematic mathematical approach to obtain the best parametric combination in order to achieve higher machining performance of WEDM process.

These types of multi-criteria decision making (MCDM) problems could be solved using well-known techniques such as grey relational analysis (GRA), artificial neural network (ANN), response surface methodology (RSM), genetic algorithm (GA) and many more. Researchers have also developed various hybrid MCDM techniques and 
applied successfully in several decision making problems. Chatterjee and Chakraborty [3] proposed a hybrid design of experiments (DoE) and technique for order preference by similarity to ideal solution (TOPSIS) methodology in developing a mathematical meta-model for the determination of technological value of cotton fiber. Also, Chakraborty and Chatterjee [4] applied the hybrid DoE-TOPSIS method for the selection of cotton fabrics. Chatterjee et al. [5] proposed a meta-model integrating DoE and evaluation based on distance from average solution (EDAS) and successfully applied to a material selection problem.

Recently, a number of literatures are also reported in parametric optimization of various NTM processes. Scott et al. [6] formulates and solves a multi-objective optimization problem in order to select the optimal parametric combination for a WEDM process. Spedding and Wang [7] presents a mathematical model describing a WEDM process using RSM and ANN considering pulse width, time between two pulses, injection set-point and wire tension as the input parameters to obtain maximum cutting speed, minimum $S R$ and surface waviness as the responses. Spedding and Wang [8] applied ANN to optimize and obtain the process parametric combinations of WEDM process through time series techniques. Sarkar et al. [9] developed a machining strategy which yields maximum process criteria in WEDM using a cascade of forward and back propagation neural network. Chakraborty and Das [10] proposed a multivariate quality loss function approach for simultaneous optimization of three NTM processes namely electro chemical machining (ECM), EDM and WEDM processes. Hewidy et al. [11] presented a mathematical model that correlates the inter-relationships of various WEDM process parameters, such as wire tension, water pressure, peak current and duty factor on $M R R, S R$ and wear ratio. However, Kung and Chiang [12] presented two mathematical models for $M R R$ and $S R$ in order to study the machinability on aluminum oxide-based ceramic material using WEDM process. They also study the effects of wire speed, pulse-on-time, peak current and duty factor on the measured responses. Yuan et al. [13] developed a multi-objective optimization technique in order to obtain the optimal parametric combination of a WEDM process considering mean pulse- on/offtime and peak current as the input parameters while considering $M R R$ and $S R$ as responses. Goswami and Kumar [14] developed a model to study the rough cut and trim cut behavior of WEDM process in order to obtain high $M R R$ and low $S R$ and wire wear ratio. Shukla and Singh [15] uses firefly algorithm (FA) in an attempt to obtain the optimal parametric combination of to two significant process, EDM and abrasive water jet machining (AWJM). Surya et al. [16] applied ANN to a WEDM process in machining of A17075 based in-situ composite to optimize the responses.

Fuzzy-logic finds its applications in various fields of research having uncertain environment. Presently, a number of multi-criteria decision making techniques integrated with fuzzy-logic have become quite popular for decision making in various fields of manufacturing. Jović et al. [17] applied adaptive neuro-fuzzy technique (ANFIS) to determine the most influencing input parameter in straight turning of mild steel (A500/A500M-13) and AISI 304 stainless steel in order to monitor the chip shapes. Julong [18] introduced grey system which emerges to be a powerful tool in the field of optimization that deals with incomplete, poor and vague data. Researchers have been effectively using grey relational technique in optimizing various multiple objectives problems in different fields of engineering $[19,20]$. The application of fuzzy logic with grey relational analysis (GRA) further improves the performance and effectiveness in solving various MCDM problems. Chakraborty et al. [21] applied grey-fuzzy logic approach in a cotton fibre selection problem. Das et al. [22] applied GRA and fuzzy logic to solve a multi- response problem of CNC milling to optimize cutting force and surface roughness. Chakraborty et al. [23] adopted grey relational analysis aided with fuzzy logic for obtaining the optimal parametric combination of three NTM processes, i.e. AWJM, ECM, and ultrasonic machining (USM) processes.

EN31 steel finds its applications in manufacturing of ball and roller bearings, beading rolls, punches, spinning tools and dies which makes it important from industrial perspective [24]. Past researchers has already attempted to obtain the optimal parametric combinations of various machining process while machining on EN31 steel. Mohanty and Nayak [25] has applied Taguchi method to optimize the $M R R$ and $S R$ as response parameters. Ugrasen et al. [26] developed a model to estimate the optimal machining performances of WEDM process using multiple regression analysis (MRA), group method data handling technique (GMDH) and ANN in machining of EN31 so as to obtain the optimal responses of accuracy, surface roughness and volumetric material removal rate. Diyaley et al. [27] has applied the combination of preference selection index (PSI) and TOPSIS to obtain the parametric combination of WEDM process while machining of EN31 steel. From the extensive review of literatures it can be concluded that, there is a keen interest among researchers to adopt different MCDM tool 
in parametric optimization of various machining process. Though, GRA has become quite popular, still it is unable to eliminate any vagueness and intangible factor present in the experimental data set. Thus, in this paper GRA aided with fuzzy logic is adopted and applied to obtain the optimal combination of process parameters so as to optimize the responses, while machining on EN31 steel using WEDM process. Four input parameters, each with three levels each is considered for experiments, while considering $M R R$ and $S R$ as the responses. ANOVA is also applied to determine the significance of each input process parameters over the machining process. Lastly, the results are verified with a confirmation test run considering the obtained optimal parametric combinations.

\section{Experimental details}

\subsection{Work material}

EN31 is a high carbon alloy steel with high hardness along with compressive strength and resistance to abrasion. Due to its high resistance against wear, EN31 finds its application in areas subject to severe wear, abrasion or high surface loading. It is mostly used in industries for the production of components like axle, roller bearings, spindle etc. Due to its poor machinability, in this paper EN31 steel is selected as the work material so as to study it machinability using WEDM process. The work specimen selected is a round bar with $14.8 \mathrm{~mm}$ diameter. EN31 is having a hardness of $63 \mathrm{HRC}$, tensile strength of $750 \mathrm{~N} / \mathrm{mm}^{2}$, modulus of elasticity of $215000 \mathrm{~N} / \mathrm{mm}^{2}$ and the chemical composition is shown in Table 1.

\subsection{Experimental setup}

The experiments are conducted using WEDM process. Brass wire having $0.25 \mathrm{~mm}$ diameter is taken as the electrode and de-ionized water as di-electric fluid are used during machining. A pictorial view of the experimental setup is provided in Fig. 1. There are a number of input parameters out of which pulse-on-time $\left(T_{o n}\right)$ (in $\left.\mu \mathrm{s}\right)$, pulse-off-time $\left(T_{\text {off }}\right)$ (in $\mu \mathrm{s}$ ), servo voltage $(S V)$ (in volts), wire tension $(W T)$ (in $\mathrm{kgf}$ ) are considered to be the variable parameters and the parameters kept constant are shown in Table 2.

$M R R$ (in $g \mathrm{~m} / \mathrm{min}$ ) and $S R$ (in $\mu \mathrm{s}$ ) are measured as the machining output parameters. $M R R$ is calculated with the difference in the weight before and after the machining process with respect to the machining time and is given by Eq. (1). Each set of combination was run for three times in order to reduce human errors. Finally, the average of the three is noted.
$M R R=\frac{m_{i}-m_{f}}{t}$

where $m_{i}$ and $m_{f}$ are the weights before and after the machining process in grams, and $t$ is time taken for machining in minutes.

$S R$ is measured using Mitutoyo Surftest J210, where the stylus of the surf test was made to run over the two machined surfaces one after the other at three different positions along the direction of lay, with the average value being considered for further analysis.

\begin{tabular}{lc}
\multicolumn{2}{c}{ Table 1 Chemical composition of EN31 steel } \\
\hline Element & Content (\%) \\
\hline Silicon oxide (SiO) & 25 \\
Chromium (Cr) & 1.46 \\
Carbon (C) & 1.08 \\
Manganese (Mn) & 0.53 \\
Nickel (Ni) & 0.33 \\
Molybdenum (Mo) & 0.06 \\
Phosphorous (P) & 0.022 \\
Sulphur (S) & 0.015 \\
Iron (Fe) & Rest \\
\hline
\end{tabular}

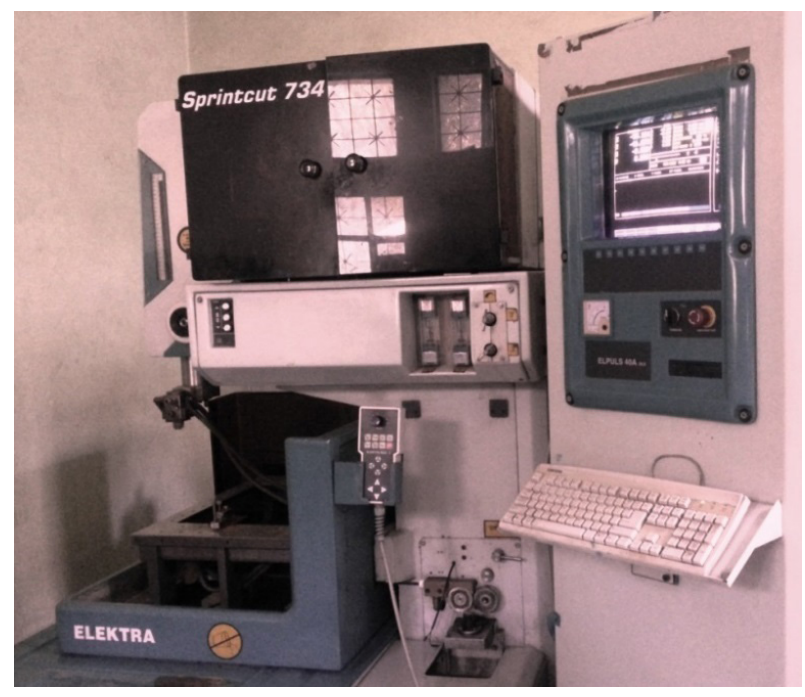

Fig. 1 Pictorial view of experimental setup

Table 2 Constant input parameters during machining

\begin{tabular}{lc}
\hline Constant parameters & Value \\
\hline Electrode & Brass wire of diameter $0.25 \mathrm{~mm}$ \\
Servo feed & $0.315 \mathrm{~m} / \mathrm{min}$ \\
Indicated power & $230 \mathrm{~mA}$ \\
Wire feed & $5-8 \mathrm{~m} / \mathrm{min}$ \\
Di-electric & De-ionized water \\
\hline
\end{tabular}




\subsection{Design of experiment by Taguchi method}

Taguchi design emerges to be an eminent approach in the field of optimization to optimize the input process parameter of a machining process based on the simulation experiments, physical experiments and experimental outputs [28]. A full factorial design plan usually considers all the possible combination of input process parameters. But, sometimes with the increased number of input parameters and their levels it is often become impossible to execute. Taguchi's orthogonal array uses a considerable subset of these combinations, exploiting the properties of fractional factorial design defining the best combination of process parameters. Thus, for a four-factor-threelevel design the maximum possible combination is $3^{4}$ or 81 experiments. In order to reduce the number of experiments $L_{9}$ orthogonal array with 9 numbers of experiments is selected with the input parameters being pulse-on-time $\left(T_{o n}\right)$, pulse-off-time $\left(T_{o f f}\right)$, servo voltage $(S V)$, and wire tension $(W T)$. The parameters with their levels selected for conducting the experiments are shown in Table 3. The range of the process parameters are so selected that they would fall within the industrially acceptable range. The output $M R R$ and $S R$ are calculated for all the 9 experiments and are shown in Table 4 respectively.

\section{Methodology}

\subsection{Grey relational analysis (GRA)}

Unlike Taguchi method, which is meant for optimizing single response optimization; the grey relational analysis can optimize multiple responses, usually conflicting in nature [29]. GRA follows the following three steps.

In the first step, the measured output parameters of $S R$ and $M R R$ are to be normalized to a range between zero and one. Normalization of the response parameters is done since the range as well as the unit of one response can differ from the others. If the characteristic of the response is of "higher-the-better", Eq. (2) is used, whereas, if the response is of "lower-the-better" characteristics, Eq. (3) is used.

$$
\begin{aligned}
& x_{i}^{*}(k)=\frac{x_{i}(k)-\min x_{i}(k)}{\max x_{i}(k)-\min x_{i}(k)}, \\
& i=1,2, \ldots, m \text { and } k=1,2, \ldots, n . \\
& x_{i}^{*}(k)=\frac{\max x_{i}(k)-x_{i}(k)}{\max x_{i}(k)-\min x_{i}(k)}
\end{aligned}
$$

where, $x_{i}(k)$ are the observed and $x_{i}^{*}(k)$ are the normalized data for the $i^{\text {th }}$ experiment and $k^{\text {th }}$ response respectively. Post normalization, the grey relational coefficient
Table 3 Machining parameters and their levels

\begin{tabular}{lccc}
\hline Control & \multicolumn{3}{c}{ Levels } \\
\cline { 2 - 4 } parameters & 1 & 2 & 3 \\
\hline$T_{\text {on }}$ & 110 & 115 & 120 \\
$T_{\text {off }}$ & 30 & 35 & 40 \\
$S V$ & 20 & 30 & 40 \\
$W T$ & 5 & 6 & 7 \\
\hline
\end{tabular}

Table 4 Experimental output performance

\begin{tabular}{lcccccc}
\hline Exp. No. & $T_{\text {on }}$ & $T_{\text {off }}$ & $S V$ & $W T$ & $M R R$ & $S R$ \\
\hline 1 & 110 & 30 & 20 & 5 & 0.05141 & 2.6 \\
2 & 110 & 35 & 30 & 6 & 0.04705 & 2.2 \\
3 & 110 & 40 & 40 & 7 & 0.05509 & 2.7 \\
4 & 115 & 30 & 30 & 7 & 0.10407 & 3.6 \\
5 & 115 & 35 & 40 & 5 & 0.1009 & 2.2 \\
6 & 115 & 40 & 20 & 6 & 0.08251 & 3.5 \\
7 & 120 & 30 & 40 & 6 & 0.09706 & 2.7 \\
8 & 120 & 35 & 20 & 5 & 0.07747 & 2.6 \\
9 & 120 & 40 & 30 & 7 & 0.05555 & 2.4 \\
\hline
\end{tabular}

$(G R C)$ for the response parameters are calculated that expresses the relationship among the ideal with the normalized data. $G R C$ value can be estimated using Eq. (4).

$\xi_{i}(k)=\frac{\Delta_{\min }+\zeta \Delta_{\max }}{\Delta_{0 i}(k)+\zeta \Delta_{\max }}$

where, $\Delta_{0 i}(k)$ is the difference between $x_{i}^{0}(k)$ and $x_{i}^{*}(k) \quad\left(x_{i}^{0}(k)\right.$ is the ideal sequence). The distinguishing coefficient $(\zeta)$ takes a value between 0 and 1 , generally $\zeta=0.5$ is preferred. It is mainly used to expand or compress the range of GRC values. $\Delta_{\text {min }}=\forall j^{\min } \in i \forall k^{\min }\left\|x_{0}(k)-x_{j}(k)\right\|$ is the smallest value of $\Delta_{0 i}$; whereas $\Delta_{\max }=\forall j{ }^{\max } \in i \forall k^{\max }\left\|x_{0}(k)-x_{j}(k)\right\|$ is the largest value of $\Delta_{0 i}$. A higher $G R C$ value for an experiment indicates that it is closer to the optimal solution with respect to a particular response.

The grey relational grade $(G R G)$ can be estimated by averaging the $G R C$ values corresponding to individual experiment and can be calculated using Eq. (5).

$\gamma_{i}=\frac{1}{n} \sum_{k=1}^{n} \xi_{i}(k)$

where, $n$ resembles the number of response parameters. The corresponding experiment number with higher value of $G R G$ indicates the input parameters for that experiment is best choice of combination among the 9 parametric combinations for the said application. 


\subsection{Fuzzy logic in grey relational analysis}

Many decision making problems are difficult to deal with, because of their inadequate information. Fuzzy set theory [30] was developed to deal with such type of decision making problems that too in an efficient way and to come up with a reasonable conclusion for these problems. It mainly converts the imprecise linguistic terms, such as highest and lowest to understandable numerical values by considering different fuzzy membership functions [31]. This theory states, "If in an environment of discourse $A$, where $\tilde{F}$ being a fuzzy subset of say $X$, can be specified by a membership function $f_{\tilde{F}}(a)$, that drafts each and every element " $a$ " in $A$ to a real number $N$ within the interval $[0,1]$. The function value $f_{\tilde{F}}(a)$ represents the grade of membership of " $a$ " in $\tilde{F}$. Larger the value of $f_{\tilde{F}}(a)$ stronger will be the grade of membership for " $a$ " in $\tilde{F}$ ".

The use of "higher-the-better" and "lower-the-better" performance characteristics in GRA produces some uncertainty within the results derived. Fuzzy logic can be effectively used in these cases in controlling these uncertainties. Integrating fuzzy logic with GRA can help in solving complex multi-response optimization [32]. The fuzzy logic system includes a fuzzifier, data base, fuzzy membership functions, rule base, fuzzy inference engine and defuzzifier. The membership functions considered for this study will be the inputs aided to the fuzzifier so as to fuzzify the input $G R C$ values which contain some amount of uncertainty with respect to the considered attributes. Then the inference engine analyses the fuzzy rules being developed, to bring out a fuzzy value as output. The defuzzifier reads the output value and finally converts the value to an understandable numerical value which is grey fuzzy reasoning grade $(G F R G)$. A fuzzy rule base consists of a set of if-then control rules which were developed that shows the inference relationship within the input $G R C$ and output $G F R G$ and can be shown as follows:

Rule 1: If $x_{1}=a_{1}, x_{2}=b_{1}, x_{3}=c_{1}$, and $x_{4}=d_{1}$,

then $G F R G=e_{1}$, else

Rule 2: If $x_{1}=a_{2}, x_{2}=b_{2}, x_{3}=c_{2}$, and $x_{4}=d_{2}$,

then $G F R G=e_{2}$, else $\ldots \ldots . \quad \ldots \ldots . \quad \ldots \ldots .$.

Rule $n$ : If $x_{1}=a_{n}, x_{2}=b_{n}, x_{3}=c_{n}$, and $x_{4}=d_{n}$, then $G F R G=e_{n}$.

where, $a_{i}, b_{i}, c_{i}$ and $d_{i}$ are the fuzzy subsets which are being defined by a membership functions, i.e. $\mu_{a i}, \mu_{b i}, \mu_{c i}$ and $\mu_{d i}$ respectively and $e_{i}$ is the grey-fuzzy output. Mamdani inference engine is normally considered which performs fuzzy reasoning with the developed rules while acknowledging max-min inference to generate a fuzzy value, $\mu_{C_{0}}(G)$.

$$
\begin{aligned}
& \mu_{C_{0}}(G)=\left(\mu_{a_{1}}\left(x_{1}\right) \wedge \mu_{b_{1}}\left(x_{2}\right) \wedge \mu_{c_{1}}\left(x_{3}\right) \wedge \mu_{d_{1}}\left(x_{4}\right) \wedge \mu_{e_{1}}(G)\right) \\
& \vee\left(\mu_{a_{2}}\left(x_{1}\right) \wedge \mu_{b_{2}}\left(x_{2}\right) \wedge \mu_{c_{2}}\left(x_{3}\right) \wedge \mu_{d_{2}}\left(x_{4}\right) \wedge \mu_{e_{2}}(G)\right) \\
& \vee \ldots \ldots \ldots \ldots \ldots . . .\left(\mu_{a_{n}}\left(x_{1}\right) \wedge \mu_{b_{n}}\left(x_{2}\right) \wedge \mu_{c_{n}}\left(x_{3}\right) \wedge \mu_{d_{n}}\left(x_{4}\right) \wedge \mu_{e_{n}}(G)\right)
\end{aligned}
$$

where, $\wedge$ and $\vee$ represents the minimum and maximum operation. Finally, while defuzzification process, the fuzzy multi-response output, $\mu_{C_{0}}(G)$ is converted to a crisp value of $G F R G\left(G_{0}\right)$.

$G_{0}=\frac{\sum G \mu_{C_{0}}(G)}{\sum \mu_{C_{0}}(G)}$.

The corresponding experiment number with the highest $G F R G$ value represents that the parametric combination of that experimental trial is the best choice when compared to the other experimental trials.

\subsection{ANOVA method}

After calculation of $G F R G$, ANOVA is applied to find out the importance of each input parameters over the machining process and their significance over the response parameters.

\section{Results and discussion}

\subsection{Grey relational analysis}

The response parameters derived from the 9 experiments are adopted to calculate the grey relational coefficients as discussed in Section 3.1. The data are initially normalized and brought to a range between 0 and 1 by using Eq. (2) in case of $M R R$ which is of "higher-the-better" characteristics and Eq. (3) in case of $S R$, which is of "lower-the-better" characteristics. The response parameters are normalized and provided in Table 8. After normalization the grey relational coefficients for each response parameters are calculated using Eq. (4) and the $G R G$ using Eq. (5) as discussed earlier and are shown in Table 5 respectively. The largest value of GRG 0.95 signifies that experiment number 5 is having the optimal combination of input parameter to give maximum $M R R$ and minimum $S R$.

The response table for grey relational grade is shown in Table 6 . These values are obtained by averaging the $G R G$ values at the corresponding level of input machining parameter. The max-min column with highest value for pulse-off-time identifies it as the most important parameter among the four input parameters. From the table, it 
Table 5 Normalized data, grey relational coefficient and grey relational grades

\begin{tabular}{lccccc}
\hline \multirow{2}{*}{ Exp. No. } & \multicolumn{2}{c}{ Normalized Data } & \multicolumn{2}{c}{$G R C$} & $G R G$ \\
& $M R R$ & $S R$ & $M R R$ & $S R$ & \\
\hline 1 & 0.0763 & 0.7143 & 0.3512 & 0.6364 & 0.4938 \\
2 & 0 & 1 & 0.3333 & 1 & 0.6667 \\
3 & 0.1412 & 0.6429 & 0.368 & 0.5834 & 0.4757 \\
4 & 1 & 0 & 1 & 0.3333 & 0.6667 \\
5 & 0.9444 & 1 & 0.8999 & 1 & 0.9500 \\
6 & 0.6217 & 0.0714 & 0.5693 & 0.35 & 0.4597 \\
7 & 0.8772 & 0.6429 & 0.8029 & 0.5834 & 0.6932 \\
8 & 0.5337 & 0.7143 & 0.5174 & 0.6364 & 0.5769 \\
9 & 0.1491 & 0.8571 & 0.3701 & 0.7777 & 0.5739 \\
\hline
\end{tabular}

Table 6 Response table for $G R G$

\begin{tabular}{lccccc}
\hline & Level 1 & Level 2 & Level 3 & Max-Min & Rank \\
\hline$T_{\text {on }}$ & 0.5454 & $\mathbf{0 . 6 9 2 1}$ & 0.6147 & 0.1467 & 3 \\
$T_{\text {off }}$ & 0.6179 & $\mathbf{0 . 7 3 1 2}$ & 0.5031 & 0.2281 & 1 \\
$S V$ & 0.5101 & 0.6358 & $\mathbf{0 . 7 0 6 3}$ & 0.1962 & 2 \\
$W T$ & $\mathbf{0 . 6 7 3 6}$ & 0.6065 & 0.5721 & 0.1015 & 4 \\
\hline
\end{tabular}

can be noted that for optimal response values of $M R R$ and $S R$, the input process parameters pulse-on-time, pulse-offtime must be maintained at level 2, while the servo voltage at level 3 and the wire tension at level 1 respectively.

The response graph has been plotted for the calculated grey relational grade and is shown in Fig. 2. From the graph it can be seen that all the three machining parameters have a grey relational grade above 0.5 . In the graph the slope of the curve for pulse-off-time is higher than the rest which indicates it to be the most influential process parameter for the considered machining process. In this graph, the symbol Ton1, Ton 2 and Ton 3 in the $x$-axis represents the three levels of pulse-on-time. Similarly the symbols Toff 1 , Toff 2 , Toff 3 represents that of pulse-offtime, $S V 1, S V 2, S V 3$ and $W T 1, W T 2, W T 3$ represents that of servo voltage and wire tension respectively. However, to improve the quality of response parameters as well as to decrease the uncertainty in the observed data, fuzzylogic is applied.

\subsection{Grey-fuzzy reasoning analysis}

The grey-fuzzy analysis is carried out in MATLAB (2013a) toolbox for generating the grey fuzzy output. In this paper, triangular membership function are considered for the two grey relational coefficients of $M R R$ and $S R$, each with five membership functions considered as lowest, low, medium, high and highest as shown in Fig. 3, while

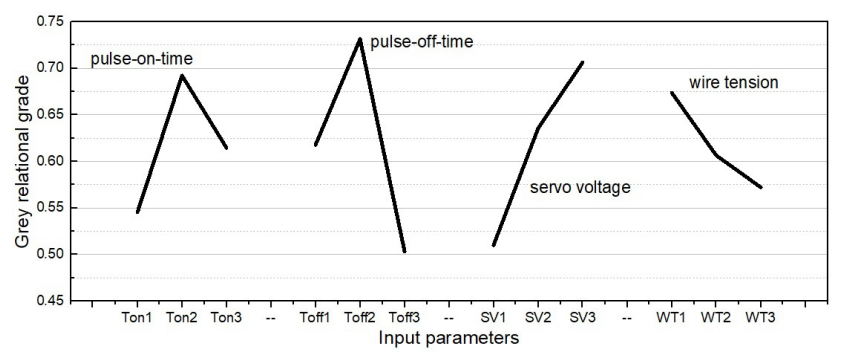

Fig. 2 Response graph for $G R G$

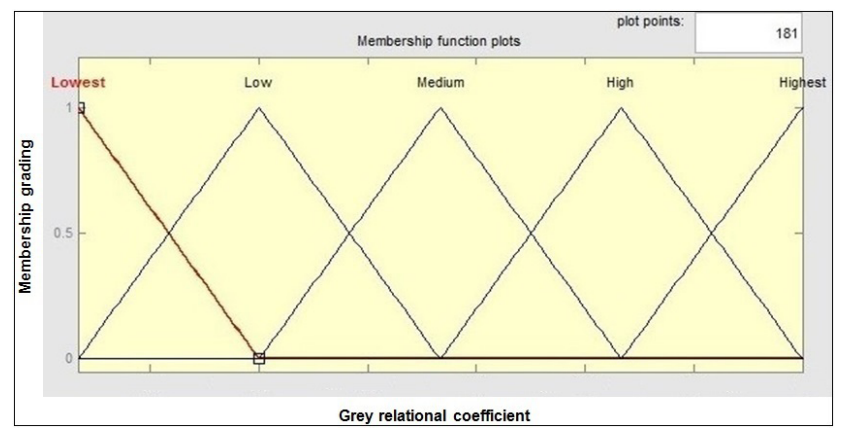

Fig. 3 Input membership functions for $M R R$ and $S R$

for the grey relational grade nine membership functions are considered as lowest, very low (VLow), low, medium low (MLow), medium, medium high (MHigh), high, very high (VHigh) and highest as shown in Fig. 4 respectively. Thus, the multi-objective parametric optimization problem becomes a two-input-one-output fuzzy logic unit with structure as presented in Fig. 5.

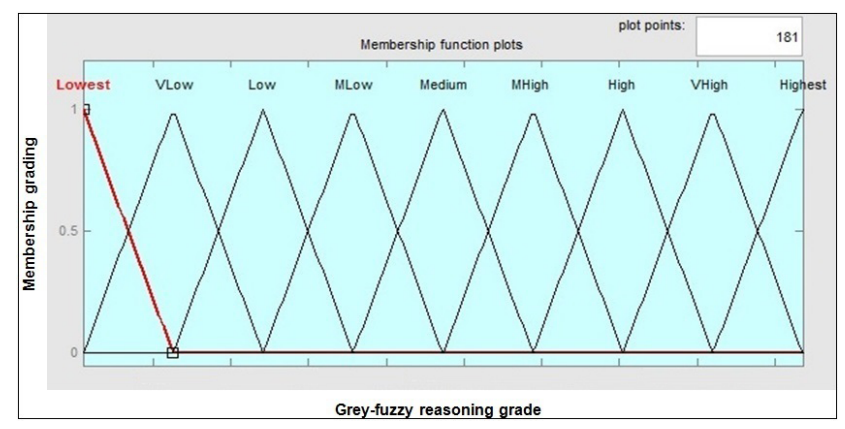

Fig. 4 Output membership functions for $G F R G$

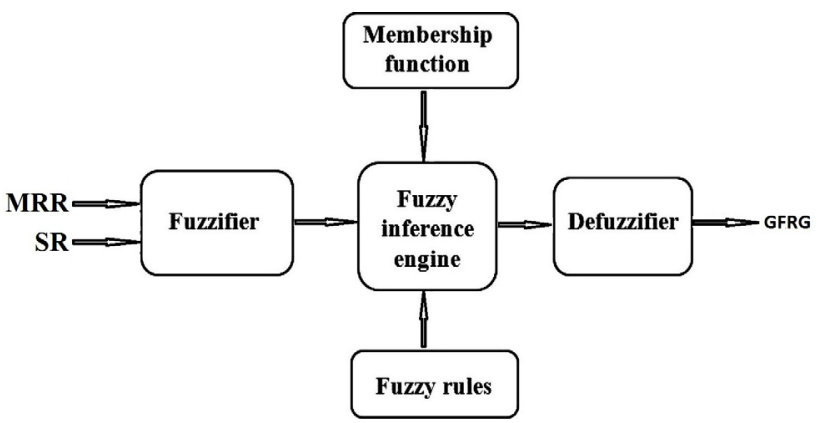

Fig. 5 Structure of two input and one output fuzzy logic 
A set of nine rules are developed representing the relation between the $G R C$ values with the $G F R G$ values in order to activate the fuzzy inference system (FIS) which are used to obtain the $G F R G$ values for all 9 experiments. The graphical representation of the nine rules developed can be seen in rule viewer as shown in Fig. 6. The three columns in the rule viewer represent two input $G R C$ values of $M R R$ and $S R$ and one output GFRG. One of such developed fuzzy rule is provided below.

If $M R R=$ Lowest and $R_{a}=$ Medium, then $G F R G=$ Lowest.

The location of each triangle in the columns in Fig. 5 indicates the decisive fuzzy set for each of the input and output values. In each triangle the height of the darkened area for that fuzzy set resembles the fuzzy membership value. From this figure it can be observed that, the input $G R C$ values of 0.3512 and 0.6364 for $M R R$ and $R_{a}$ respectively for the first experiment results in defuzzied $G F R G$ value of 0.508. In the same way, for all the 9 experiments the GFRG values are computed, as shown in Table 7. From the table, it can be confirmed that the experiment number 5 has the highest value of GFRG which indicates it to have the best optimal parametric combination that gives maximum $M R R$ and minimum $S R$.

Table 8 represents the response table for GFRG. These values are calculated by averaging the corresponding GFRG value of each input parameters. The maxmin column signifies that the servo voltage is the most influencing input parameters followed by pulse-off-time among the four parameters. It was found that the optimal combination obtained from Table 8 is the same as that obtained in Table 6 which confirms that to obtain the best response values of $M R R$ and $S R$, the input parameters pulse-on-time, pulse-off-time must be maintained at

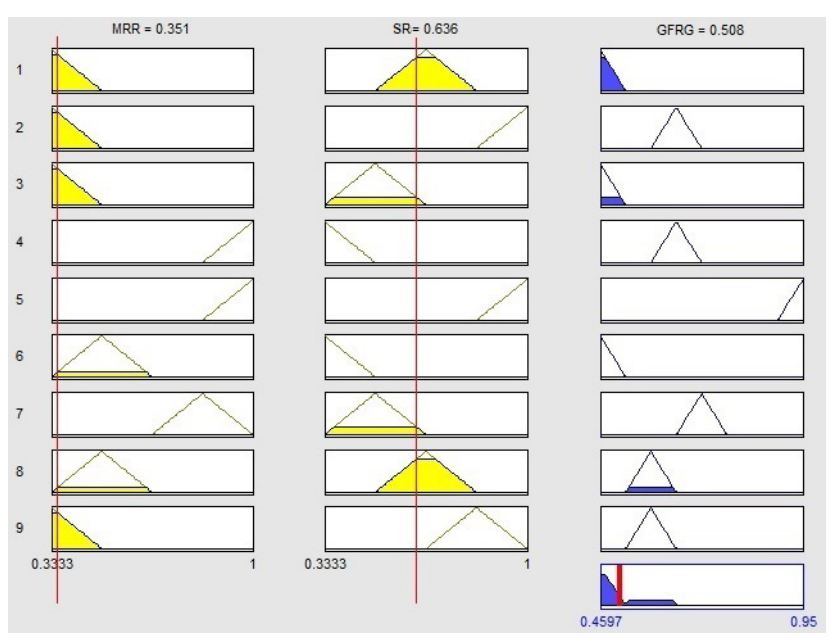

Fig. 6 Rule viewer
Table 7 Grey-fuzzy reasoning grade

\begin{tabular}{ll}
\hline Exp. No. & GFRG \\
\hline 1 & 0.508 \\
2 & 0.644 \\
3 & 0.531 \\
4 & 0.644 \\
5 & 0.926 \\
6 & 0.481 \\
7 & 0.705 \\
8 & 0.582 \\
9 & 0.558 \\
\hline
\end{tabular}

Table 8 Response table for $G F R G$

\begin{tabular}{lccccc}
\hline & Level 1 & Level 2 & Level 3 & Max-Min & Rank \\
\hline$T_{\text {on }}$ & 0.5610 & $\mathbf{0 . 6 8 3 7}$ & 0.6150 & 0.1227 & 3 \\
$T_{\text {off }}$ & 0.6190 & $\mathbf{0 . 7 1 7 3}$ & 0.5233 & 0.1940 & 2 \\
$S V$ & 0.5237 & 0.6153 & $\mathbf{0 . 7 2 0 7}$ & 0.1970 & 1 \\
$W T$ & $\mathbf{0 . 6 7 2 0}$ & 0.6100 & 0.5777 & 0.0943 & 4 \\
\hline
\end{tabular}

level 2, while the servo voltage at level 3 and the wire tension at level 1. Fig. 7 shows the response graph plot for the calculated $G F R G$ which shows that all the three machining input parameters have a $G F R G$ value above 0.5 . In the graph as shown in Fig. 6, the slope of the curve for pulseoff-time is higher than the rest which indicates it to be the most influential parameter for the machining process.

\subsection{Analysis of variance (ANOVA)}

To understand the importance of each input process parameters over the responses, the $G F R G$ obtained are subjected to ANOVA process. The role of each input factor on the multiple performance characteristics can be analysed using ANOVA which is done at $95 \%$ confidence. Fisher's $f$-test is adopted to find out the change in which the machining process parameter holds a significant effect on the multiple performance characteristics. Larger $f$-value and smaller $p$-value signifies that the change of that process parameter holds a stronger influence on the response parameters. ANOVA is applied to the results obtained from grey fuzzy

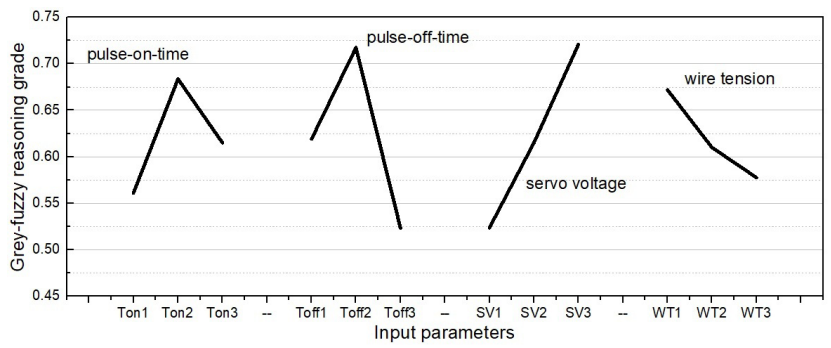

Fig. 7 Response graph for $G F R G$ 
reasoning analysis and are tabulated in Table 9. It can be seen from the table that the degrees of freedom (DoF) for residual error comes zero as it doesn't have enough data. Normally this happens if 4 input parameters with 3 levels are considered for experiments with $L_{9}$ orthogonal array for analysis. Hence the need for ANOVA pooling arises.

ANOVA pooling is a usual practice of revising and reestimating the result in order to neglect a factor which is of very less significant. It is done by relating the insignificant factor with the residual error. Pooling is mainly considered because of two reasons. Firstly, when more number of factors is considered for an experiment, it is probable that half of those factors would be more influential than the others. Secondly, statistical predictions encounter two mistakes one being alpha and the other being beta. Alpha mistakes happens when we call something is important though it is not. Beta mistake is the adverse in which significant factors are unknowingly ignored. If a factor fails the test of significance it is needed to be pooled. It is mandatory for the DoF of residual error to have a nonzero value in order to perform the test of significance. Pooling starts with the factors having less influence on the response parameters. Here, pulse-off-time is seen to be the least influencing factor; hence it is pooled as shown in Table 10.

From the ANOVA table it is observed that the pulse-ontime, servo voltage and wire tension has $p$-value less than 0.5 which confirms these parameters to be statistically significant and thus have a positive contribution in determining GFRG. It can be also concluded that servo voltage is the most influencing parameter as it has the highest $f$-value

Table 9 Analysis of variance for GFRG (before pooling)

\begin{tabular}{lcccccc}
\hline Source & DoF & Seq SS & Adj SS & Adj MS & $f$-value & $p$-value \\
\hline$T_{\text {on }}$ & 2 & 0.0227 & 0.0227 & 0.0113 & $*$ & $*$ \\
$T_{\text {off }}$ & 2 & 0.0565 & 0.0004 & 0.0002 & $*$ & $*$ \\
$S V$ & 2 & 0.0583 & 0.0642 & 0.0321 & $*$ & $*$ \\
$W T$ & 2 & 0.0096 & 0.0096 & 0.0048 & $*$ & $*$ \\
Error & 0 & $*$ & $*$ & $*$ & & \\
Total & 8 & & & & & \\
\hline
\end{tabular}

Table 10 Analysis of variance for GFRG (after pooling)

\begin{tabular}{lcccccc}
\hline Source & DoF & Seq SS & Adj SS & Adj MS & $f$-value & $p$-value \\
\hline$T_{\text {on }}$ & 2 & 0.0227 & 0.0227 & 0.0113 & 53.78 & 0.018 \\
$S V$ & 2 & 0.0583 & 0.1102 & 0.0551 & 261.33 & 0.004 \\
$W T$ & 2 & 0.0657 & 0.0657 & 0.0328 & 155.75 & 0.006 \\
Error & 2 & 0.0004 & 0.0004 & 0.0002 & & \\
Total & 8 & 0.1471 & & & & \\
\hline
\end{tabular}

and least $p$-value in compared to others, followed by wire tension and pulse-on-time.

In order to define the relationship between the input machining parameters and the obtained GFRG, the following regression equation is developed. Based on the regression model the corresponding surface plots are developed presented in Fig. 8 which also supports the above observations.

$G F R G=11.9232-0.139081 \times T_{\text {on }}-0.383945 \times T_{\text {off }}$

$+0.109619 \times S V+0.605071 \times W T+0.00451429$

$\times T_{\text {on }} \times T_{\text {off }}-0.02005 \times T_{\text {off }} \times W T$.

Existing well known techniques are available that can be effectively applied in obtaining the parametric combination of WEDM process. However, in GRA, the derived results are solely depend upon the original data set, and is easy to calculate and simple to apprehend, and is flexible to deal with several types of MCDM problems. In addition to that the distinguishing coefficient $(\zeta)$, in Eq. (3), can be selected based upon a decision maker's judgment. Moreover, the adoption of fuzzy logic with GRA eliminates any vagueness and intangible factor present in the experimental data set, thus making it one of the best MCDM approach.

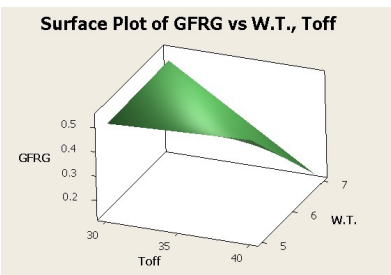

(a)

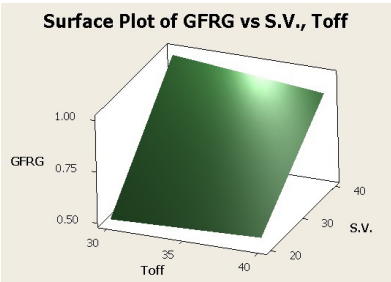

(c)

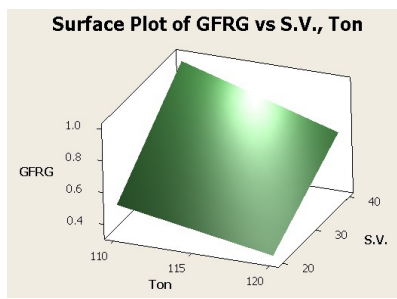

(e)

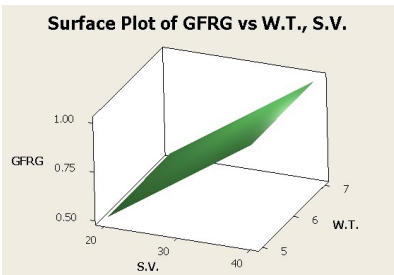

(b)

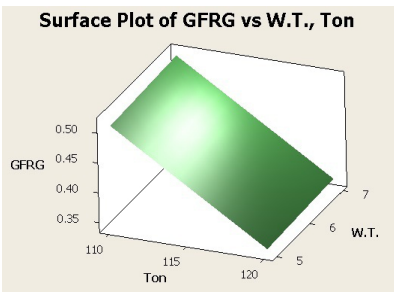

(d)

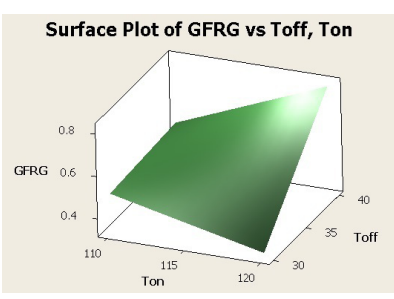

(f)
Fig. 8 Surface plots showing the effects of different WEDM process parameters on $G F R G$ value 


\subsection{Confirmation test}

A confirmation test is performed so as to check the enhancement in the quality of response values. The predicted GFRG can be calculated using Eq. (10).

$G_{p}=G_{p}+\sum_{i=1}^{N}\left(\bar{G}_{i}-G_{m}\right)$

where, $G_{p}$ is the predicted $G F R G, G_{m}$ is the mean $G F R G$ for the 9 experiments, $\bar{G}_{i}$ is the mean $G F R G$ of the corresponding optimal $i^{\text {th }}$ response and $N$ is the total number of input parameters.

The confirmation experiment done with the same experimental setup reveals that the $M R R$ is increased from $0.0514 \mathrm{gm} / \mathrm{min}$ to $0.1065 \mathrm{gm} / \mathrm{min}, S R$ reduces from $2.6 \mu \mathrm{m}$ to $2.3 \mu \mathrm{m}$ as shown in Table 11. Thus the obtained input parametric combinations improve the $G F R G$ from 0.508 to 0.9240 , which equals to $81.89 \%$ of improvement.

\section{Conclusion}

In this present work, machining of EN31 steel is carried out with four machining input parameters as pulseon-time, pulse-off-time, servo voltage and wire tension, and the response parameters as MRR and $S R$ in WEDM process. Taguchi's $L_{9}$ orthogonal array is used for design of experiments to perform machining operation on the work material. It was found that pulse-on-time of $115 \mu \mathrm{s}$,

\section{References}

[1] Jain, V. K. "Advanced Machining Processes", 4th ed., Allied Publishers Pvt. Limited, New Delhi, India, 2005.

[2] Ho, K. H., Newman, S. T., Rahimifard, S., Allen, R. D. "State of the art in wire electrical discharge machining (WEDM)", International Journal of Machine Tools and Manufacture, 44(1213), pp. 1247-1259, 2004.

https://doi.org/10.1016/j.ijmachtools.2004.04.017

[3] Chatterjee, P., Chakraborty, S. "Development of a meta-model for the determination of technological value of cotton fiber using design of experiments and the TOPSIS method", Journal of Natural Fibers, 15(6), pp. 882-895, 2018.

https://doi.org/10.1080/15440478.2017.1376303

[4] Chakraborty, S. and Chatterjee, P. "A Developed Meta-model for Selection of Cotton Fabrics Using Design of Experiments and TOPSIS Method", Journal of The Institution of Engineers (India): Series E, 98(2), pp. 79-90, 2017.

https://doi.org/10.1007/s40034-017-0108-X

[5] Chatterjee, P., Banerjee, A., Mondal, S., Boral, S., Chakraborty, S. "Development of a Hybrid Meta-Model for Material Selection Using Design of Experiments and EDAS Method", Engineering Transactions, 66(2), pp. 187-207, 2018.
Table 11 Comparison table for initial and optimal parametric combination

\begin{tabular}{|c|c|c|c|}
\hline \multirow{3}{*}{ Levels } & \multirow{3}{*}{ 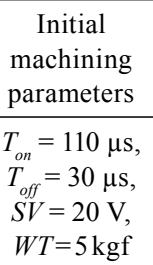 } & \multicolumn{2}{|c|}{$\begin{array}{l}\text { Optimum machining } \\
\text { parameters }\end{array}$} \\
\hline & & \multicolumn{2}{|c|}{$\begin{array}{c}T_{\text {on }}=115 \mu \mathrm{s}, T_{\text {off }}=35 \mu \mathrm{s} \\
S V=40 \mathrm{~V}, W T=5 \mathrm{kgf}\end{array}$} \\
\hline & & Predicted & Experimental \\
\hline$M R R(\mathrm{gm} / \mathrm{min})$ & 0.0514 & - & 0.1009 \\
\hline$S R(\mu \mathrm{m})$ & 2.6 & - & 2.2 \\
\hline$G F R G$ & 0.508 & 0.9340 & 0.9260 \\
\hline $\begin{array}{l}\text { Improvement in } \\
G F R G\end{array}$ & - & 0.4260 & 0.4180 \\
\hline$\%$ improvement & - & $83.86 \%$ & $82.28 \%$ \\
\hline
\end{tabular}

pulse-off-time of $35 \mu \mathrm{s}$, servo voltage of $40 \mathrm{~V}$ and wire tension of $5 \mathrm{kgf}$ is the optimal combination for the input machining parameters. ANOVA results admit that servo voltage is the most influencing parameter which adversely affects the response parameters. The adopted approach is quite simple and easy to apprehend, and is unaffected with respect to any additional parameter, eliminating any vagueness and intangible factor present in the experimental data set. Moreover, the developed surface plot will help a process engineer to easily identity a desired parametric combination as per the requirements.

[6] Scott, D., Boyina, S., Rajurkar, K. P. "Analysis and optimization of parameter combinations in wire electrical discharge machining", International Journal of Production Research, 29(11), pp. 21892207, 1991.

https://doi.org/10.1080/00207549108948078

[7] Spedding, T. A., Wang, Z. Q. "Parametric optimization and surface characterization of wire electrical discharge machining process", Precision Engineering, 20(1), pp. 5-15, 1997.

https://doi.org/10.1016/S0141-6359(97)00003-2

[8] Spedding, T. A., Wang, Z. Q. "Study on modeling of wire EDM process", Journal of Materials Processing Technology, 69(1-3), pp. 18-28, 1997.

https://doi.org/10.1016/S0924-0136(96)00033-7

[9] Sarkar, S., Mitra, S., Bhattacharyya, B. "Wire electrical discharge machining of gamma titanium aluminide for optimum process criteria yield in single pass cutting operation", International Journal of Manufacturing Technology and Management, 7(2-4), pp. 207223,2005 .

https://doi.org/10.1504/IJMTM.2005.006831

[10] Chakraborty, S., Das, P. P. "A multivariate quality loss function approach for parametric optimization of non-traditional machining processes", Management Science Letters, 8(8), pp. 873-884, 2018. https://doi.org/10.5267/j.msl.2018.6.001 
[11] Hewidy, M. S., El-Taweel, T. A., El-Safty, M. F. "Modelling the machining parameters of wire electrical discharge machining of Inconel 601 using RSM", Journal of Materials Processing Technology, 169(2), pp. 328-336, 2005. https://doi.org/10.1016/j.jmatprotec.2005.04.078

[12] Kung, K.-Y., Chiang, K.-T."Modeling and Analysis of Machinability Evaluation in the Wire Electrical Discharge Machining (WEDM) Process of Aluminum Oxide-Based Ceramic", Materials and Manufacturing Processes, 23(3), pp. 241-250, 2008. https://doi.org/10.1080/10426910701860616

[13] Yuan, J., Wang, K., Yu, T., Fang, M. "Reliable multi-objective optimization of high-speed WEDM process based on Gaussian process regression", International Journal of Machine Tools and Manufacture, 48(1), pp. 47-60, 2008. https://doi.org/10.1016/j.ijmachtools.2007.07.011

[14] Goswami, A., Kumar, J. "Trim cut machining and surface integrity analysis of Nimonic 80A alloy using wire cut EDM", Engineering Science and Technology, an International Journal, 20(1), pp. 175186, 2017. https://doi.org/10.1016/j.jestch.2016.09.016

[15] Shukla, R., Singh, D. "Selection of parameters for advanced machining processes using firefly algorithm", Engineering Science and Technology, an International Journal, 20(1), pp. 212-221, 2017. https://doi.org/10.1016/j.jestch.2016.06.001

[16] Surya, V. R., Kumar, K. M. V., Keshavamurthy, R., Ugrasen, G., Ravindra, H. V. "Prediction of Machining Characteristics using Artificial Neural Network in Wire EDM of A17075 based In-situ Composite", Materials Today: Proceedings, 4(2), pp. 203-212, 2017.

https://doi.org/10.1016/j.matpr.2017.01.014

[17] Jović, S., Arsić, N., Vukojević, V., Anicic, O., Vujičić, S. "Determination of the important machining parameters on the chip shape classification by adaptive neuro-fuzzy technique", Precision Engineering, 48, pp. 18-23, 2017. https://doi.org/10.1016/j.precisioneng.2016.11.001

[18] Julong, D. "Introduction to Grey System Theory", The Journal of Grey System, 1(1), pp. 1-24, 1989.

[19] Reddy, V. C., Deepthi, N., Jayakrishna, N. "Multiple Response Optimization of Wire EDM on Aluminium HE30 by using Grey Relational Analysis", Materials Today: Proceedings, 2(4-5), pp. $2548-2554,2015$. https://doi.org/10.1016/j.matpr.2015.07.201

[20] Singh, P. N., Raghukandan, K., Pai, B. C. "Optimization by Grey relational analysis of EDM parameters on machining Al-10\% $\mathrm{SiC}_{\mathrm{p}}$ composites", Journal of Materials Processing Technology, 155-156, pp. 1658-1661, 2004.

https://doi.org/10.1016/j.jmatprotec.2004.04.322

[21] Chakraborty, S., Das, P. P., Kumar, V. "A Grey Fuzzy Logic Approach for Cotton Fibre Selection", Journal of The Institution of Engineers (India): Series E, 98(1), pp. 1-9, 2017. https://doi.org/10.1007/s40034-017-0099-7
[22] Das, B., Roy, S., Rai, R. N., Saha, S. C. "Application of grey fuzzy logic for the optimization of CNC milling parameters for $\mathrm{Al}-4.5 \% \mathrm{Cu}-\mathrm{TiC} \mathrm{MMCs}$ with multi-performance characteristics", Engineering Science and Technology, an International Journal, 19(2), pp. 857-865, 2016.

https://doi.org/10.1016/j.jestch.2015.12.002

[23] Chakraborty, S., Das, P. P., Kumar, V. "Application of grey-fuzzy logic technique for parametric optimization of non-traditional machining processes", Grey Systems: Theory and Application, 8(1), pp. 46-68, 2018. https://doi.org/10.1108/GS-08-2017-0028

[24] Valera, H. Y., Bhavsar, S. N. "Experimental Investigation of Surface Roughness and Power Consumption in Turning Operation of EN 31 Alloy Steel", Procedia Technology, 14, pp. 528-534, 2014. https://doi.org/10.1016/j.protcy.2014.08.067

[25] Mohanty, D., Nayak, N. C. "Effect of Process Parameters on Performance of EN-31 Steel using WEDM: Experimentation and Optimization", International Journal of Engineering Research and Technology, 5(7), pp. 536-547, 2016.

[26] Ugrasen, G., Ravindra, H. V., Prakash, G. V. N., Keshavamurthy, R. "Estimation of Machining Performances Using MRA, GMDH and Artificial Neural Network in Wire EDM of EN-31", Procedia Materials Science, 6, pp. 1788-1797, 2014.

https://doi.org/10.1016/j.mspro.2014.07.209

[27] Diyaley, S., Shilal, P., Shivakoti, I., Ghadai, R. K., Kalita, K. "PSI and TOPSIS Based Selection of Process Parameters in WEDM", Periodica Polytechnica Mechanical Engineering, 61(4), pp. 255260, 2017.

https://doi.org/10.3311/PPme.10431

[28] Krishnaiah, K., Shahabudeen, P. "Applied design of experiments and Taguchi methods", 1st ed., PHI Learning Pvt. Ltd., New Delhi, India, 2012.

[29] Kurt, M., Hartomacioğlu, S., Mutlu, B., Köklü, U. "Minimization of the surface roughness and form error on the milling of free-form surfaces using a grey relational analysis", Materiali in tehnologije / Materials and Technology, 46(3), pp. 205-213, 2012.

[30] Zadeh, L. A. "Fuzzy sets", Information and Control, 8(3), pp. 338353, 1965. https://doi.org/10.1016/S0019-9958(65)90241-X

[31] Dewangan, S., Gangopadhyay, S., Biswas, C. K. "Multi-response optimization of surface integrity characteristics of EDM process using grey-fuzzy logic-based hybrid approach", Engineering Science and Technology, an International Journal, 18(3), pp. 361368, 2015. https://doi.org/10.1016/j.jestch.2015.01.009

[32] Pradhan, V., Das, P. P. "Parametric optimization of CNC turning on glass-fibre-reinforced plastic (GFRP) pipes: A grey-fuzzy logic approach", IOP Conference Series: Materials Science and Engineering, 377(1), 012180, 2018. https://doi.org/10.1088/1757-899X/377/1/012180 